\title{
Surgical Management of a Rare Case of Clinodactyly with Delta Phalanx in a Pediatric Patient
}

\section{ISSN: 2576-8875}

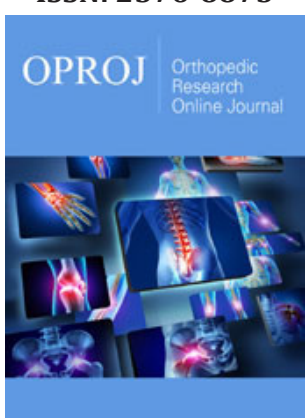

*Corresponding author: Rolando Willacy, Howard University Hospital, Department of Orthopaedic Sugery, USA

Submission: 海 March 15, 2021

Published: 阱April 05, 2021

Volume 8 - Issue 2

How to cite this article: Rolanda Willacy, DianneMarie Omire-Mayor, Henok Tesfay, Julencia Curtis, Jamil Williams, Robert Wilson. Surgical Management of a Rare Case of Clinodactyly with Delta Phalanx in a Pediatric Patient. Ortho Res Online J. 8(2). OPROJ. 000681. 2021. DOI: 10.31031/OPROJ.2021.08.000681

Copyright@: Rolanda Willacy, This article is distributed under the terms of the Creative Commons Attribution 4.0 International License, which permits unrestricted use and redistribution provided that the original author and source are credited.

\author{
Rolanda Willacy ${ }^{1 *}$, DianneMarie Omire-Mayor ${ }^{2}$, Henok Tesfay ${ }^{2}$, Julencia \\ Curtis $^{2}$, Jamil Williams ${ }^{2}$ and Robert Wilson ${ }^{1,2}$
}

${ }^{1}$ Howard University Hospital, Department of Orthopaedic Sugery, USA

${ }^{2}$ Howard University College of Medicine, USA

\begin{abstract}
Clinodactyly is a rare congenital birth defect that can result in radial or ulnar deviation of the fingers. One form of clindactyl is delta phalanx. This condition does not cause loss of function unless the deviation is severe. Clinodactyly with delta phalanx can be distinguished by other soft tissue disorder with radiographs. However, in the case of early development MRI is indicated because the cartilaginous epiphysis has not ossified and therefore cannot be visualized by radiographs. In this case, a 19 month old patient presents with a left index finger deformity that was managed surgically. The most common form of early surgical intervention of the delta phalanx is physiolysis. This procedure is recommended for newborns and infants because more correction of angulation is needed with more growth of the phalanx. Therefore, early intervention is indicated in these cases.
\end{abstract}

\section{Introduction}

Clinodactyly is a rare congenital birth defect that results in radial but also, less commonly, ulnar deviation of the fingers. This typically occurs in the small finger. One form of clinodactyly is a delta phalanx, which presents as a C-shaped epiphysis bracketing. Clinodactyly with delta phalanx is commonly inherited in an autosomal dominant manner and can have variable expressivity [1]. This defect can be observed most commonly in the middle phalanx, but also in the proximal phalanx or the metacarpal [2]. In delta phalanx, the primary ossification center, diaphysis and metaphysysis, are bracketed by a secondary ossification center [3]. This was first characterized by Jones in 1964 as a delta phalanx but was later characterized by Light and Ogden as a "longitudinal epiphyseal bracket" [4]. They was noted that there were separate primary and distal secondary ossification centers extended toward each other in the epiphyseal cartilage, bracketing the entirety of the primary ossification center [5].

Clinodactyly generally doesn't cause loss of function unless the deviation is severe, the disorder is primarily diagnosed from finding deviation of one or more digits in the radioulnar plane. Clinodactyly caused by delta phalanx can be distinguished from other forms caused by soft tissue or secondary ossification using radiography methods. Radiographs demonstrate the merging of the proximal and distal epiphysis and the specific C-shaped or trapezoidshaped phalanx. Precise angle of deviation is also measured radiographically during the diagnosis and monitoring progress after surgical intervention [2]. However, the use of radiographs is limited in pediatrics patients to minimize unnecessary exposure. In the case of early development, before the cartilaginous epiphysis is ossified, radiographs are unable to show delta phalanx. As a result, MRI is the recommended diagnostic test [6]. MRI also removes the risk of unnecessary radiation exposure to young patients. Finally, though there is familial origin of some clinodactyly, genetic testing hasn't been suggested as a diagnosis.

\section{Case Report}

A 19 month old male was referred to the Pediatric Hand and Upper Extremity Clinic for the evaluation of a left index finger deformity. The parents reported that the patient was born with the deformity, which caused no discomfort, pain or limitations in function. However, it was noted that the patient demonstrated some difficulty in grasping and picking up objects with the left hand. Their primary concern was that the deformity would progress and worsen, 
and they were interested in discussing the option of surgical intervention. They were unable to obtain radiographs during the clinical visit, due to insurance authorization issues. Physical examination revealed a deformity of the middle phalanx of the index finger, with ulnar-sided deviation of the distal phalanx. No swelling or tenderness to palpation was noted. The patient had full range of motion at the Metacarpal Phalangeal (MP) and Proximal Interphalangeal (PIP) joints, with limitedability to flex the Distal Interphalangeal (DIP) joint.

The Orthopaedic Surgeon discussed with the parents of the patient that the deformity was likelyclinodactyly, with delta phalanx as the probable cause. Radiographs were obtained to confirm the diagnosis. A follow-up visit was scheduled, and radiographs were reviewed with the patients. The recommendation was made for surgical management, and the parents agreed with the plan.

On the date of the surgery, the patient was placed under general anesthesia in the supine position. A mid-axial incision was made down to the level of the middle phalanx, which had a trapezoidal shape. C-arm images and gross photos were obtained before the start of the procedure (Figure 1). The osteotomy site was then opened, and cancellous and cortical bone allograft was then placed, and fixation was maintained with the use of a Kirschner wire (Figure 2) The incision was then closed, and a sterile dressing was applied with a protective splint. The patient returned with his parents for a follow-up appointment to check the site of the pin and radiographs demonstrated that fixation was maintained. During the following visit, the pin was removed. The patient was in no acute distress, and the incision site was intact. There was marked improvement of the curvature of the left index finger. A follow up appointment was scheduled to continue to observe the progress of the graft incorporating with the bone.

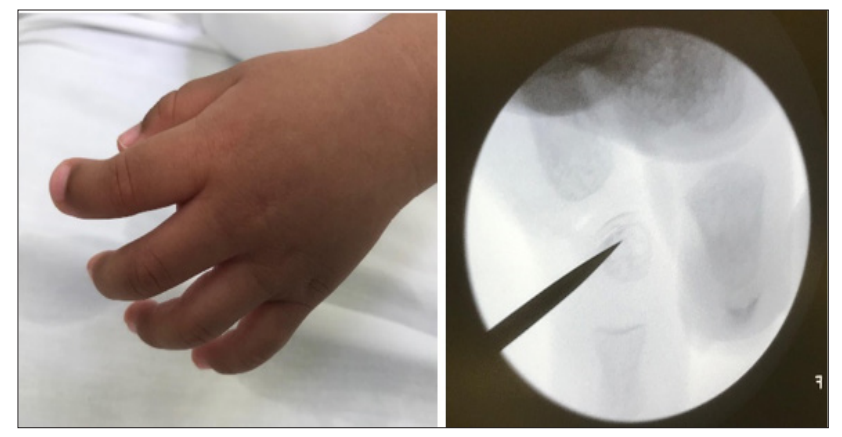

Figure 1: (a) Gross image of clinodactyly with delta phalanx of the left index finger prior to the start of the procedure

(b) Intraoperative fluoroscopic image of the delta phalanx of the left index finger
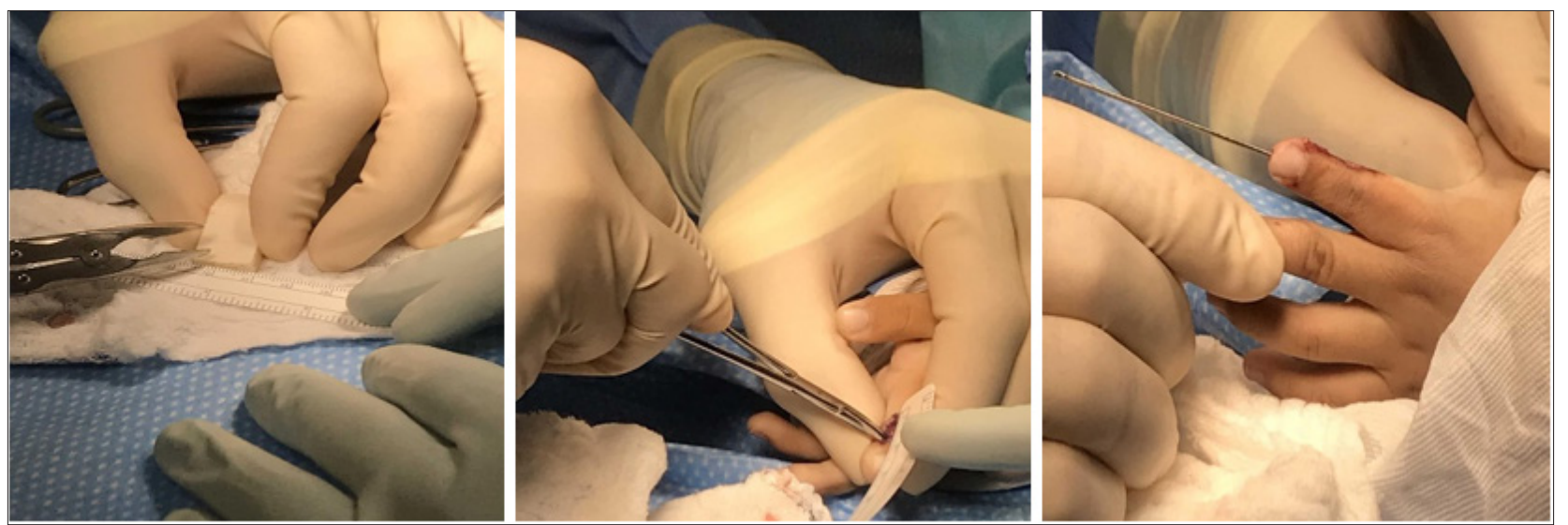

Figure 2:

a. Preparation of the cancellous and cortical bone allograft

b. Placement of the allograft

c. Fixation maintained with a Kirschner wire.

\section{Conclusion}

There are many different classifications of clinodactyly, and there different forms of surgical management to correct this deformity. In patients with milder delta phalanx deformities, many do not experience functional limitations and therefore surgery is delayed until the child patient is at least 6 years of age and is mainly done for cosmetic reasons [7]. However, in more severe cases surgical intervention may be indicated earlier. Surgical procedures include opening wedge osteotomy; closing wedge osteotomy; reverse wedge osteotomy; and resection of the abnormalepiphysis with interposition of an H-graft or fat graft [7].
The most common form of early surgical intervention of the delta phalanx is physiolysis. This procedure is recommended for newborns and infants because more correction of angulation is needed with more growth of the phalanx. Therefore, early intervention is indicated in these cases [8]. Physiolysis is also a simple technique with little to no difficulty for the child and corrects the deformity slowly over a period of years [9]. Surgical procedures done at older ages, such as wedge osteotomies, pose the risk of shortening of the digit and may requires a secondary procedure and fixation of the bone. Therefore, wedge osteotomies are more successful in older children who can be managed better 
post-operatively. Although different procedures are recommended depending on the age of the patient and severity of the deformity, all procedures require long term follow-up to ensure that the correction is maintained.

This case demonstrated an acceptable correction of the delta phalanx with an osteotomy of themiddle phalanx and use of bone allograft in a pediatric patient. While there remains some variability in treatment strategies for delta phalanx, early physiolysis has been shown to achieve optimum growth of the digits with minimal deformity [8]. The timing of the physiolysis also impacts the need for an inter positional graft in the space vacated by the excised pathological epiphysis. Comparative analysis of clinical and radiographic appearance of these deviated digits allows for early excision of the epiphysis with minimal difficulty for the child [2]. Appropriate and early identification of a delta phalanx in pediatric patients is important in surgical planning to achieve maximal function and cosmetic appearance of the involved digit [6]. Optimal results have been observed in children who have the surgery prior to 6 years of age, with long term follow-up required to demonstrate procedural efficacy [10]. There are studies that indicate that if correction is not obtained earlier in development, corrective osteotomy may be required later [1]. However, it has been noted that attempting repeat physiolysis is not recommended initially given the minimal benefit and risk of premature physical fusion; delayed wedge osteotomy can safely follow physiolysis to gain further correction [1]. Future retrospective studies should be conducted to examine older patients with correction of clinodactyly with delta phalanx, and their postoperative radiographic and functional outcomes.

\section{References}

1. Albright S, Xue A, Koshy J, Robert C, Hollier LH (2011) Bilateral proximal delta phalanges: An unusual presentation of familial congenital clinodactyly. Hand 6(3): 340-343.

2. Medina JA, Lorea P, Elliot D, Foucher G (2016) Correction of clinodactyly by early physiolysis: 6-year results. J Hand Surg Am 41(6): e123-127.

3. Mubarak S, O'brien T, Davids J (1993) Metatarsal epiphyseal bracket: Treatment by central physiolysis. J Pediatr Orthop 13(1): 5-8.

4. Jones GB (1964) Delta phalanx. The Journal of Bone and Joint Surgery 46-B(2): 226-228.

5. Light TR, Ogden JA (1981) The longitudinal epiphyseal bracket: Implications for surgical correction. J Pediatr Orthop 1(3): 299-305.

6. Johnson JM, Higgins TJ, Lemos D (2011) Appearance of the delta phalanx (longitudinally bracketed epiphysis) with MR imaging. Pediatric Radiology 41: 394-396.

7. Ravishanker R, Bath AS (2004) Distraction-A minimally invasive technique for treating camptodactyly and clinodactyly. Med J Armed Forces India 60(3): 227-230.

8. Alexander D, Mubarak S (2013) Longitudinal epiphyseal bracket. J Child Orthop 7(6): 449-454.

9. Caouette L, Caroline L, Patricia E, Constantin S (2002) Physiolysis for correction of clinodactyly in children. J Hand Surg Am 27(4): 659-665.

10. Iba K, Wada T, Yamashita T (2015) Correction of thumb angulations after physiolysis of delta phalanges in a child with Rubinstein-Taybi syndrome: A case report. Case Reports Plast Surg Hand Surg 2(1): 12-14. 